\section{Efectos de la violencia y la discriminación en la salud mental de bisexuales, lesbianas y homosexuales de la Ciudad de México}

\author{
Effects of violence and discrimination \\ on the mental health of bisexuals, lesbians, \\ and gays in Mexico City
}

Luis Ortiz-Hernández ${ }^{1}$

María Isabel García Torres 1

\footnotetext{
1 Departamento

de Atención a la Salud, Universidad Autónoma Metropolitana Xochimilco, Coyoacán, México.

Correspondência L. Ortiz-Hernández Departamento de Atención a la Salud, Universidad Autónoma Metropolitana Xochimilco. Calzada del Hueso 1100, Col. Villa Quietud, Coyoacán, México 04960. lortiz@correo.xoc.uam.mx
}

\begin{abstract}
The objectives of this study were to estimate the prevalence of suicidal ideation, suicide attempts, mental disorders, and alcoholism in bisexuals, lesbians, and gays in Mexico City and analyze the possible relationship between violence, discrimination, and the mental health of these population groups. A questionnaire was administered to 506 bisexuals, lesbians, and gays attending support organizations and institutions. Eight forms of discrimination and fourteen forms of violence based on sexual orientation were researched. The study found the following prevalence rates: $39.0 \%$ suicidal ideation, 15.0\% suicide attempts, $27.0 \%$ mental disorders, and $18.0 \%$ alcoholism. Prevalence of alcoholism was $21.0 \%$ among bisexual and lesbian women, which is higher than in other women. Discrimination was correlated with suicide attempts and mental disorders, whereas violence was a risk factor for suicidal ideation, suicide attempts, mental disorders, and alcoholism. Violence and to a lesser extent discrimination suffered by bisexuals, lesbians, and gays are associated with psychological distress, and policies are needed to counter this situation.
\end{abstract}

Violence; Prejudice; Mental Health; Homosexuality

\section{Introducción}

En la vida cotidiana los bisexuales, las lesbianas y los homosexuales se enfrentan a diferentes formas de prejuicio, además de que existen pocas organizaciones que atiendan los problemas específicos de esta población y no hay reconocimiento institucional y legal de los distintos arreglos familiares entre individuos del mismo sexo. Las formas más evidentes de violencia van desde los insultos verbales y las agresiones físicas, hasta formas extremas como los asesinatos. La Comisión Ciudadana Contra los Crímenes de Odio por Homofobia $(\mathrm{CCCOH})^{1}$ ha documentado, a partir de una revisión en periódicos publicados en México, que entre 1995 y 2000 ocurrieron 213 asesinatos contra homosexuales y lesbianas, los cuales se han caracterizado por la brutalidad y violencia extrema con la que han sido realizados. La CCCOH sugiere que por cada caso que ella ha documentado existen por lo menos otros tres. A parte del trabajo de la $\mathrm{CCCOH}$, en México han existido pocos intentos de documentar las distintas formas de agresión que sufren los bisexuales, las lesbianas y los homosexuales y sus repercusiones en la salud mental de éstos.

La mayoría de los estudios en los bisexuales, las lesbianas y los homosexuales sobre la relación entre opresión y daños a la salud se han realizado en países industrializados, como Estados Unidos de Norteamérica, Europa y Aus- 
tralia 2,3. En América Latina los estudios sobre salud en los que se han incluido la población bisexual, lesbiana y homosexual básicamente se han centrado en el análisis del comportamiento de la infección de VIH/SIDA en hombres que tienen sexo con otros hombres, pero no se han evaluado otros problemas de salud que son frecuentes en esta población. A pesar de que cada vez más académicos y políticos de la región se preocupan por esta problemática, aún es incipiente el conocimiento que se tiene sobre las condiciones en las que se desenvuelve la vida de los bisexuales, las lesbianas y los homosexuales. Aunque los resultados de las investigaciones realizadas en países industrializados deben considerarse, es necesario reconocer que las condiciones de Latinoamérica son diferentes por la mayor desigualdad socioeconómica y la mayor rigidez en la asignación y contenido de los estereotipos de género 4 .

La población bisexual, lesbiana y homosexual se enfrenta a diferentes formas de prejuicio y exclusión social, entre las que se encuentran la violencia interpersonal y la discriminación. La violencia interpersonal ha sido definida como: "el uso deliberado de la fuerza física o el poder, ya sea en grado de amenaza o efectivo, contra uno mismo, otra persona o un grupo o comunidad, que cause o tenga muchas probabilidades de causar lesiones, muerte, daños psicológicos, trastornos del desarrollo o privaciones" 5 (p. 5). La violencia interpersonal cubre un amplio abanico de actos y comportamientos que van desde la violencia física, sexual y psíquica hasta las privaciones y el abandono 5 . Llama la atención que el documento no habla sobre violencia en minorías o grupos como los bisexuales, las lesbianas y los homosexuales, menciona la violencia sexual en contra de mujeres y hombres, pero no específica en concreto las repercusiones que pueden tener las personas que por su orientación sexual experimentan este tipo de agresiones.

Desde la perspectiva de la salud, la violencia puede ser conceptualizada como causa y efecto. La violencia es causa porque es una forma de opresión, pero también es un efecto, ya que la violencia es en sí misma un daño a la salud en tanto que altera la integridad psicológica y física de los individuos. Es evidente que la violencia física tiene consecuencias inmediatas y visibles que van desde lesiones leves hasta el homicidio. Sin embargo, la violencia dirigida hacia los bisexuales, las lesbianas y los homosexuales (o violencia por odio) difiere de la violencia "cotidiana". La violencia por odio son acciones con las que se intenta dañar o intimidar a las personas debido a su raza, etnia, orien- tación sexual u otro estatus de grupo minoritario 6 . La relevancia de distinguir a la violencia por odio es que, si bien todas las formas de violencia tienen efecto en las víctimas, la violencia por odio representa un caso especial debido a que tiene mayor impacto tanto en la víctima que la sufre como en el grupo social al que pertenece la víctima 7 . Los bisexuales, las lesbianas y los homosexuales pueden ser particularmente vulnerables a los efectos psicológicos negativos de la violencia por odio debido a que: (a) la mayoría de la población sigue teniendo una actitud negativa hacia los bisexuales, las lesbianas y los homosexuales, lo que hace que aquellos que son víctimas de violencia pueden llegar a creer que ellos se merecen esa agresión, (b) los bisexuales, las lesbianas y los homosexuales que consideran que su orientación sexual es negativa, es decir, que tienen homofobia internalizada, pueden aceptar esta noción, lo cual puede incrementar su distrés psicológico después de ser víctima de una agresión, (c) debido a que la identidad de los bisexuales, las lesbianas y los homosexuales con frecuencia se desarrolla al margen de la familia y la comunidad de origen, los miembros de esta población no disfrutan automáticamente del apoyo de la familia y la comunidad cuando ellos son víctimas de violencia y (d) es poco probable que ellos aprendan en la niñez estrategias para afrontar las distintas formas que adopta el prejuicio contra la homosexualidad 7 .

En la discriminación se niega el acceso a oportunidades, recursos y servicios a los individuos debido a su orientación. La discriminación tiene como consecuencia una limitación en el desarrollo de las potencialidades de los individuos ya que éstos no tienen acceso a las oportunidades, los recursos y los servicios. Por ejemplo, en el ámbito de la atención médica, los profesionales de la salud tienen actitudes homofóbicas, lo cual puede provocar que los bisexuales, las lesbianas y los homosexuales no busquen información y no accedan a los servicios médicos 8 . Además la discriminación, al igual que la violencia, refuerza la vivencia de la homosexualidad como una condición negativa. La discriminación puede ser originada por los prejuicios que los prestadores de servicios han aprendido en sus familias y el medio social en general. En el caso de los profesionales que proporcionan servicios (e.g. profesores, médicos, enfermeras y psicólogos), es frecuente que sean formados en instituciones educativas donde se enseña que la homosexualidad es una enfermedad o una forma de inadaptación social, o bien, en sus planes de estudio no se contemplan las necesidades de está población. La dis- 
criminación también puede originarse por que las empresas e instituciones tienen políticas que excluyen a los bisexuales, las lesbianas y los homosexuales. A pesar de que teóricamente 7 la violencia y la discriminación que reciben los bisexuales, las lesbianas y los homosexuales pueden tener efectos sobre su salud mental, existe poca evidencia empírica al respecto 9,10,11.

Considerando lo anterior, los objetivos de este estudio fueron: (a) estimar la frecuencia de daños a la salud mental en los bisexuales, las lesbianas y los homosexuales de la Ciudad de México y (b) analizar la posible relación de la violencia y la discriminación con la salud mental de dicha población.

\section{Material y métodos}

Se realizó un estudio observacional, transversal y analítico. Se ha reconocido que es prácticamente imposible hacer una muestra representativa de la población de bisexuales, lesbianas y homosexuales, debido a que no se cuenta con un marco de muestras y a que su orientación sexual está estigmatizada lo que impide que muchos bisexuales, lesbianas y homosexuales comuniquen con veracidad su orientación sexual, por lo que se ha recomendado que cuando se estudien muestras por conveniencia de bisexuales, lesbianas y homosexuales se trate de seleccionar a individuos con características heterogéneas 12. Dicha recomendación fue llevada a cabo en nuestro estudio, puesto que se aplicó un cuestionario a 506 bisexuales, lesbianas y homosexuales que acudieron a organizaciones de distinta naturaleza en las cuales se esperaba acudieran diversos "sub-grupos" de bisexuales, lesbianas y homosexuales. También se llegó a estos espacios, ya que tienen la ventaja de que se puede contactar a un número considerable de bisexuales, lesbianas y homosexuales bajo condiciones de seguridad, además de que brindan condiciones óptimas (e.g. ambiente sin presiones y sin ruido) para aplicar la encuesta, condiciones que no se encuentran en, por ejemplo, discotecas, bares y baños públicos. Entre septiembre y noviembre del 2001 se acudió a organizaciones e instituciones que atienden a los bisexuales, las lesbianas y los homosexuales y que se encontraban en la Ciudad de México, incluyéndose a un grupo de activismo, nueve grupos de socialización, dos instituciones religiosas, una clínica de VIH/ SIDA, un café, un centro de atención psicológica y una escuela de artes en el nivel de bachillerato. Sin embargo, aplicar las encuestas en los centros mencionados tiene el inconve- niente de que por lo regular asisten individuos pertenecientes a estratos socioeconómicos medios y altos, así como sujetos con un grado avanzado de auto-aceptación de su homosexualidad. Por ello, una parte la población fue contactada por medio de redes personales del autor y por una lista de correos electrónicos de un grupo de socialización.

El presente proyecto puede ser ubicado, considerando el reglamento de la Ley General de Salud en materia de investigación para la salud, como una investigación sin riesgo en tanto que no se realizó " ninguna intervención o modificación intencionada en las variables fisiológicas, psicológicas y sociales de los individuos que participan en el estudio" (artículo 17), por lo cual no fue indispensable que los sujetos que participaron en el proyecto proporcionaran su consentimiento informado por escrito (artículo 23) 13.

La aplicación del cuestionario la realizaron cuatro pasantes de la licenciatura en psicología social y el coordinador del proyecto (L. O. H.). Para promover que los encuestados proporcionaran información veraz, antes de que llenaran el cuestionario se les explicaban las dos formas en que se mantendría su identidad en anonimato: (a) en el formato del cuestionario no se solicitó ningún dato que permitiera identificar al encuestado (e.g. nombre, domicilio, teléfono, etc.) y (b) una vez que terminaban de responder el cuestionario lo introducían en una caja donde se revolvía con el resto de los cuestionarios. El último procedimiento no fue posible aplicar en los cuestionarios aplicados a conocidos por redes personales y a los que respondieron mediante correo electrónico.

Considerando la propuesta de Klein 14 para evaluar la orientación sexual de los entrevistados se les preguntó cual era el sexo de las personas por las que se sentían sexualmente atraídos. La pregunta que se aplicó fue: ¿Usted por quién se siente sexualmente atraído?, las respuestas eran: (a) Sólo por mujeres, (b) Principalmente por mujeres, pero no sólo por ellas, (c) Por mujeres y hombres, pero más por mujeres, (d) Por hombres y mujeres por igual, (e) Por hombres y mujeres, pero más por hombres, (f) Principalmente por hombres, pero no sólo por ellos, (g) Sólo por hombres y (h) No está seguro. Las respuestas fueron agrupadas de la siguiente manera:

- Bisexual-heterosexual: para los varones incluye las opciones $\mathrm{B}$ y C, en las mujeres las opciones E y F;

- Bisexual-homosexual: para los varones incluye las opciones D, E y F, en las mujeres abarca B, C y D; 
- Homosexual: para los varones incluye la opción $\mathrm{G}$ y en las mujeres la A.

Para averiguar la existencia de discriminación por ser bisexual, lesbiana u homosexual algunos autores 9,10 han preguntado directamente a los entrevistados si han sido objeto de discriminación. Este procedimiento puede ser incorrecto, puesto que cada entrevistado puede interpretar de forma distinta la palabra "discriminación". Por eso se formularon 8 preguntas con las que se indaga si los entrevistados se han enfrentado a situaciones concretas que pueden reflejar la existencia de discriminación debido a su homosexualidad. Con estas preguntas se pretende evaluar la existencia de discriminación en la obtención de vivienda, en el trabajo, la escuela y el acceso y uso de servicios.

Los daños a la salud que se investigaron fueron seleccionados porque en investigaciones anteriores se ha observado que son frecuentes en los bisexuales, las lesbianas y los homosexuales 2,3. Mediante una pregunta se valoró la percepción que tenían los entrevistados sobre su estado general de salud, para el análisis se formaron dos grupos: (a) buena y muy buena y (b) muy mala, mala y regular. Para evaluar la existencia de ideación e intento de suicidio en el cuestionario se incluyeron las preguntas utilizadas en la versión A del cuestionario de la Encuesta Nacional de Adicciones 15. En el caso de la pregunta de intento de suicidio, las respuestas fueron dicotomizadas a no (nunca) y sí (una vez, dos o tres veces, cuatro o cinco veces y seis o más veces).

Para identificar sintomatología indicativa de trastornos mentales comunes se utilizó la versión corta de 28 reactivos del Cuestionario General de Salud (CGS) validada en estudiantes universitarios de México 16. Se utilizó un sistema de puntuación 0-1, es decir, a las dos primeras opciones de respuesta se les otorgó un valor de 0 y a las dos últimas se les dio un puntaje de 1, este esquema de puntuación fue sugerido en el estudio original del CGS 17. Posteriormente, se sumaron los puntajes de las 28 preguntas y se utilizó un punto de corte de 5/6 16 , en otras palabras, los individuos con una puntuación total de cinco o menos fueron considerados como normales y los que tuvieron un puntaje total de seis o más fueron calificados como "casos potenciales" de trastornos mentales comunes.

El alcoholismo fue identificado mediante la escala Alcohol Use Disorder Identification Test (AUDIT), que consiste en diez preguntas sobre la cantidad y la frecuencia de consumo de alcohol, síntomas de dependencia y problemas relacionados con su consumo ${ }^{18}$. En las prime- ras ocho preguntas de la escala, a las opciones de respuesta se les asignó un valor de 0 (opción a) a 4 (opción e); mientras que en las dos últimas preguntas a las opciones de respuesta se les asignó valores de 0,2 y 4, respectivamente. A continuación se obtuvo la puntuación total de la escala y las personas que tuvieron una puntuación de 8 o más fueron considerados como casos probables de alcoholismo, individuos con puntuaciones inferiores a ese límite fueron clasificados como "normales" 18.

Para estudiar la asociación de la violencia y la discriminación con los daños a la salud se calcularon razones de prevalencias e intervalos de confianza al 95\%. Para ello primero se estimaron, mediante regresiones logísticas, razones de momios con sus respectivos intervalos de confianza al 95\%. En los modelos de regresión se incorporaron las variables edad, sexo y escolaridad, ya que podían actuar como confusores. Debido a que el estudio consistió en una encuesta transversal se transformaron las razones de momios (RM) en razones de prevalencias (RP) mediante la siguiente fórmula:

$\mathrm{RP}=\mathrm{RM} /\left[\left(1-\mathrm{P}_{0}\right)+\left(\mathrm{P}_{0} \bullet \mathrm{RM}\right)\right]$,

donde $\mathrm{P}_{0}$ es la prevalencia ajustada en el grupo de referencia, con la misma ecuación se estimaron los intervalos de confianza para las RP 19. El análisis estadístico se realizó en el programa SPSS versión 10.

\section{Resultados}

En la Tabla 1 se muestran las características sociodemográficas de la población estudiada. Se encuestaron a 318 varones bisexuales y homosexuales y 188 mujeres bisexuales y lesbianas. El promedio de edad en ambos sexos fue de 29 años. Con relación a las mujeres, en los varones fue más alto el porcentaje de solteros sin pareja (74,0\% versus 55,0\%), pero más baja la proporción de personas con pareja (10,0-13,0\% y $18,0-19,0 \%$, respectivamente). En los varones bisexuales y homosexuales, el 41,0\% contaba con bachillerato y el $40,0 \%$ con licenciatura y más, en las mujeres bisexuales y lesbianas los porcentajes fueron de $35,0 \%$ y $42,0 \%$, respectivamente; no existieron diferencias entre los sexos con relación a esta variable. Aunque tanto en hombres como en mujeres la mayoría se sentía sexualmente atraído por personas del mismo sexo (69,0\% y 52,0\%, respectivamente), en las últimas dicho porcentaje fue menor, en ellas fueron más altas las proporciones de personas que se sentían atraídas tanto por hombres como mujeres o que no estaban seguras de por quienes sentían atracción sexual. 
Características sociodemográficas de la población.

\begin{tabular}{|c|c|c|c|c|c|}
\hline & Total & Varones & Mujeres & $t / \chi^{2}$ & $p$ \\
\hline N & 506 & 318 & 188 & & \\
\hline \multicolumn{6}{|l|}{ Edad (años) } \\
\hline Promedio & 29 & 29 & 29 & 0,87 & 0,382 \\
\hline Mínima & 13 & 13 & 15 & & \\
\hline Máxima & 70 & 70 & 53 & & \\
\hline \multicolumn{6}{|l|}{ Estado civil (\%) } \\
\hline Soltero, sin pareja & 67 & 74 & 55 & 22,74 & $<0,000$ \\
\hline Con pareja & 15 & 13 & 18 & & \\
\hline Con pareja y cohabitan & 14 & 10 & 19 & & \\
\hline Otros & 5 & 3 & 9 & & \\
\hline \multicolumn{6}{|l|}{ Escolaridad (\%) } \\
\hline Primaria & 4 & 4 & 3 & 1,98 & 0,576 \\
\hline Secundaria & 18 & 19 & 17 & & \\
\hline Bachillerato & 37 & 35 & 41 & & \\
\hline Licenciatura y posgrado & 41 & 42 & 39 & & \\
\hline \multicolumn{6}{|l|}{ Atracción sexual (\%)* } \\
\hline Bisexual-heterosexual & 5 & 3 & 9 & 22,21 & $<0,000$ \\
\hline Bisexual-homosexual & 30 & 28 & 35 & & \\
\hline Homosexual & 63 & 69 & 52 & & \\
\hline No está seguro & 2 & 1 & 4 & & \\
\hline
\end{tabular}

* Ver en el texto la explicación de cada categoría.

En la Tabla 2 se muestra la frecuencia con la que los entrevistados han sido objeto de distintas formas de discriminación y violencia interpersonal, así como de los daños a la salud. Las formas más frecuentes de discriminación fueron: no los contrataron en un empleo $(13,0 \%)$, amenaza, extorsión y detención por policías $(11,0 \%)$ y maltrato por parte de empleados $(10,0 \%)$, no existiendo diferencias entre los sexos. Las formas de violencia más frecuentes fueron: ofensas verbales $(32,0 \%)$, acoso sexual (18,0\%), "molestaron sexualmente" (15,0\%), asalto $(12,0 \%)$, siguieron o persiguieron $(12,0 \%) \mathrm{y}$ amenazas verbales $(11,0 \%)$. Entre los varones bisexuales y homosexuales existió una mayor proporción de victimas de ofensas verbales, mientras que las mujeres con más frecuencia sufrieron agresión física. Respecto a los indicadores del estado de salud, la mayoría percibía que su estado de salud era bueno o muy bueno $(76,0 \%)$. Cuatro de cada diez entrevistados informaron ideación suicida y poco más de una quinta parte $(25,0 \%)$ comunicó haber intentado suicidarse. Un tercio de la población presentó sintomatología indicativa de trastornos mentales comunes y una quinta parte presentó riesgo de tener alcoholismo. En las mujeres fueron más altas las prevalencias de intento de suicidio y trastornos mentales comunes.

En la Tabla 3 se muestra la asociación de la discriminación en el último año con los daños a la salud. La discriminación dentro del trabajo se asoció con un incremento en el riesgo de presentar intento de suicidio y trastornos mentales comunes, mientras que la discriminación en la obtención de vivienda aumentó la probabilidad de experimentar trastornos mentales comunes. El maltrato por empleados de servicios se asoció con el intento de suicidio. Ninguna de las formas de discriminación se asoció con la percepción del estado de salud, la ideación suicida y el alcoholismo.

La relación de los daños a la salud con la violencia se presenta en la Tabla 4 . La violencia verbal se asoció con los trastornos mentales comunes. La violencia física y el observar cómo agredían a un conocido se relacionaron con mayor riesgo de ideación suicida, intento de suicidio, trastornos mentales comunes y alcoholismo. El acoso sexual incrementó la probabilidad de presentar intento de suicidio, mien- 
Frecuencia de violencia, discriminación y daños a la salud.

\begin{tabular}{|c|c|c|c|c|c|}
\hline & Total & Hombres & Mujeres & $\chi^{2}$ & $p$ \\
\hline \multicolumn{6}{|l|}{ Discriminación } \\
\hline No lo han contratado en empleo & 13 & 14 & 12 & 0,77 & 0,379 \\
\hline Dentro de trabajo & 8 & 7 & 11 & 2,14 & 0,143 \\
\hline En obtención de vivienda & 6 & 7 & 6 & 0,22 & 0,638 \\
\hline Han negado ingreso a escuela & 8 & 8 & 7 & 0,08 & 0,769 \\
\hline Dentro de escuela & 4 & 3 & 6 & 2,84 & 0,092 \\
\hline Han negado acceso a otros servicios & 5 & 4 & 7 & 1,93 & 0,164 \\
\hline Maltrato de empleados de servicios & 10 & 9 & 11 & 0,19 & 0,661 \\
\hline De policías & 11 & 12 & 8 & 1,71 & 0,190 \\
\hline \multicolumn{6}{|l|}{ Violencia } \\
\hline Ofensas verbales & 32 & 35 & 26 & 3,61 & 0,057 \\
\hline Amenazas verbales & 11 & 10 & 12 & 0,55 & 0,456 \\
\hline Arrojaron algún objeto & 7 & 7 & 8 & 0,01 & 0,896 \\
\hline Escupieron & 2 & 1 & 4 & 3,47 & 0,062 \\
\hline Siguieron o persiguieron & 12 & 11 & 12 & 0,04 & 0,838 \\
\hline Agresión física & 7 & 5 & 10 & 4,76 & 0,029 \\
\hline Lesión física con arma & 3 & 2 & 4 & 1,65 & 0,198 \\
\hline Acoso sexual & 18 & 20 & 17 & 0,72 & 0,396 \\
\hline Molestaron sexualmente* & 15 & 16 & 13 & 1,04 & 0,306 \\
\hline Violación sexual & 3 & 3 & 3 & 0,22 & 0,673 \\
\hline Daño de objetos personales & 9 & 7 & 11 & 2,42 & 0,119 \\
\hline Asalto & 12 & 12 & 10 & 0,35 & 0,551 \\
\hline Robo & 6 & 7 & 5 & 1,13 & 0,287 \\
\hline Golpearon/asesinaron conoc. & 5 & 5 & 5 & 0,06 & 0,803 \\
\hline \multicolumn{6}{|l|}{ Salud percibida } \\
\hline Muy mala & 1 & 1 & 2 & 5,57 & 0,233 \\
\hline Mala & 2 & 1 & 3 & & \\
\hline Regular & 21 & 20 & 22 & & \\
\hline Buena & 51 & 50 & 52 & & \\
\hline Muy buena & 25 & 28 & 21 & & \\
\hline Ideación suicida & 39 & 36 & 44 & 2,65 & 0,103 \\
\hline \multicolumn{6}{|l|}{ Intento de suicidio } \\
\hline Nunca & 85 & 88 & 79 & 12,94 & 0,012 \\
\hline $1 \mathrm{vez}$ & 9 & 6 & 13 & & \\
\hline 2 ó 3 veces & 3 & 2 & 5 & & \\
\hline 4 ó 5 veces & 2 & 2 & 3 & & \\
\hline 6 o más veces & 1 & 2 & 0 & & \\
\hline Trastornos mentales comunes & 27 & 23 & 33 & 5,40 & 0,020 \\
\hline Alcoholismo & 18 & 17 & 21 & 1,04 & 0,307 \\
\hline
\end{tabular}

* Incluye besos, caricias y manoseo.

Fuente: encuestas aplicadas a asistentes de organizaciones e instituciones de, o enfocadas a, bisexuales, lesbianas y homosexuales, Ciudad de México, 2001 
Asociación* de la discriminación en el último año con daños en la salud.

\begin{tabular}{|c|c|c|c|c|c|c|c|c|c|c|}
\hline & \multicolumn{2}{|c|}{$\begin{array}{l}\text { Estado de salud } \\
\text { regular o malo }\end{array}$} & \multicolumn{2}{|c|}{ Ideación suicida } & \multicolumn{2}{|c|}{$\begin{array}{l}\text { Intento de } \\
\text { suicidio }\end{array}$} & \multicolumn{2}{|c|}{$\begin{array}{l}\text { Trastornos mentales } \\
\text { comunes }\end{array}$} & \multicolumn{2}{|c|}{ Alcoholismo } \\
\hline & $\mathrm{RP}$ & IC95\% & $\mathrm{RP}$ & IC95\% & $\mathrm{RP}$ & IC95\% & $\mathrm{RP}$ & IC95\% & $\mathrm{RP}$ & IC95\% \\
\hline \multicolumn{11}{|c|}{ No lo han contratado en empleo } \\
\hline No & $(22,4)$ & & $(38,5)$ & & $(14,2)$ & & $(25,9)$ & & $(18,6)$ & \\
\hline Sí & 1,27 & $0,81-1,85$ & 1,09 & $0,78-1,43$ & 1,59 & $0,93-2,52$ & 1,26 & $0,83-1,78$ & 1,00 & $0,56-1,66$ \\
\hline \multicolumn{11}{|c|}{ Discriminación dentro de trabajo } \\
\hline No & $(23,1)$ & & $(38,5)$ & & $(14,5)$ & & $(25,4)$ & & $(18,3)$ & \\
\hline Sí & 0,98 & $0,53-1,66$ & 1,24 & $0,84-1,66$ & 1,88 & $1,02-3,09$ & 1,67 & $1,08-2,32$ & 1,29 & $0,68-2,19$ \\
\hline \multicolumn{11}{|c|}{$\begin{array}{l}\text { Discriminación en obtención } \\
\text { de vivienda }\end{array}$} \\
\hline No & $(23,1)$ & & $(40,6)$ & & $(14,9)$ & & $(25,8)$ & & $(18,0)$ & \\
\hline Sí & 1,01 & $0,51-1,78$ & 0,52 & $0,25-0,97$ & 1,77 & $0,86-3,12$ & 1,70 & $1,05-2,41$ & 1,73 & $0,93-2,80$ \\
\hline \multicolumn{11}{|c|}{ Han negado ingreso a escuela } \\
\hline No & $(23,9)$ & & $(38,8)$ & & $(14,2)$ & & $(25,7)$ & & $(18,1)$ & \\
\hline Sí & 0,63 & $0,28-1,28$ & 0,94 & $0,58-1,37$ & 1,54 & $0,80-2,67$ & 1,39 & $0,84-2,05$ & 1,11 & $0,57-1,98$ \\
\hline \multicolumn{11}{|c|}{ Discriminación dentro de escuela } \\
\hline No & $(23,5)$ & & $(39,0)$ & & $(14,9)$ & & $(26,3)$ & & $(18,3)$ & \\
\hline Sí & 0,60 & $0,19-1,57$ & 1,14 & $0,61-1,73$ & 1,89 & $0,80-3,55$ & 1,46 & $0,73-2,35$ & 1,56 & $0,68-2,89$ \\
\hline \multicolumn{11}{|c|}{ Han negado otros servicios } \\
\hline No & $(22,6)$ & & $(38,9)$ & & $(14,9)$ & & $(26,3)$ & & $(18,0)$ & \\
\hline Sí & 1,55 & $0,80-2,52$ & 1,10 & $0,62-1,63$ & 1,60 & $0,69-3,09$ & 1,34 & $0,71-2,15$ & 1,77 & $0,88-2,97$ \\
\hline \multicolumn{11}{|c|}{ Maltrato empleados de servicios } \\
\hline No & $(23,2)$ & & $(37,6)$ & & $(14,0)$ & & $(25,6)$ & & $(17,9)$ & \\
\hline Sí & 0,99 & $0,55-1,64$ & 1,37 & $0,97-1,76$ & 1,81 & $1,02-2,93$ & 1,40 & $0,89-20,00$ & 1,30 & $0,73-2,12$ \\
\hline \multicolumn{11}{|c|}{ Discriminación de policías } \\
\hline No & $(23,5)$ & & $(39,0)$ & & $(14,7)$ & & $(26,3)$ & & $(18,0)$ & \\
\hline Sí & 0,88 & $0,49-1,48$ & 0,98 & $0,66-1,36$ & 1,36 & $0,73-2,31$ & 1,15 & $0,72-1,71$ & 1,26 & $0,71-2,05$ \\
\hline
\end{tabular}

* Se informan razones de prevalencias ajustadas por edad-escolaridad y sexo con sus respectivos intervalos de confianza al $95 \%$.

Las cifras entre paréntesis son las prevalencias ajustadas en el grupo de referencia.

Fuente: encuestas aplicadas a asistentes de organizaciones e instituciones de, o enfocadas a, bisexuales, lesbianas

y homosexuales, Ciudad de México, 2001.

tras que la violencia sexual mostró ser un factor de riesgo para la ideación suicida y el intento de suicidio. Las personas que habían sufrido daño, asalto o robo de propiedades presentaron mayor riesgo de intento de suicidio, trastornos mentales comunes y alcoholismo. Ninguna de las formas de violencia se relacionó con la percepción del estado de salud.

\section{Discusión}

Antes de comentar los resultados del estudio es pertinente señalar sus limitaciones. Cuando se estudian muestras elegidas por conveniencia, como en esta investigación, existe una probabilidad elevada de que uno o varios segmentos de la población de interés estén sub- o sobrerepresentados, situación que ocasiona que los hallazgos observados en este tipo de muestras no sean extrapolables a la población blanco. Por ello, los resultados de este estudio proporcionan una aproximación a la situación de los individuos que acuden a las instituciones y organizaciones que visitaron. No obstante, es muy probable que los bisexuales, las lesbianas y los homosexuales que no asisten a las instituciones y organizaciones en las que se aplicó el cues- 


\begin{tabular}{|c|c|c|c|c|c|c|c|c|c|c|}
\hline & \multicolumn{2}{|c|}{$\begin{array}{l}\text { Estado de salud } \\
\text { regular o malo }\end{array}$} & \multicolumn{2}{|c|}{ Ideación suicida } & \multicolumn{2}{|c|}{$\begin{array}{l}\text { Intento de } \\
\text { suicidio }\end{array}$} & \multicolumn{2}{|c|}{$\begin{array}{c}\text { Trastornos mentales } \\
\text { comunes }\end{array}$} & \multicolumn{2}{|c|}{ Alcoholismo } \\
\hline & $\mathrm{RP}$ & IC95\% & $\mathrm{RP}$ & IC95\% & $\mathrm{RP}$ & IC95\% & $\mathrm{RP}$ & IC95\% & $\mathrm{RP}$ & IC95\% \\
\hline \multicolumn{11}{|l|}{ Violencia verbal } \\
\hline No & $(23,3)$ & & $(34,6)$ & & $(12,2)$ & & $(21,4)$ & & $(16,2)$ & \\
\hline Sí & 0,98 & $0,66-1,38$ & 1,26 & $0,99-1,56$ & 1,49 & $0,93-2,26$ & 1,64 & $1,20-2,13$ & 1,21 & $0,79-1,79$ \\
\hline Violencia física & 1 & & & & & & & & & \\
\hline No & $(21,8)$ & & $(36,5)$ & & $(13,3)$ & & $(22,9)$ & & $(16,3)$ & \\
\hline Sí & 1,24 & $0,81-1,79$ & 1,38 & $1,05-1,71$ & 1,74 & $1,07-2,66$ & 1,83 & $1,33-2,38$ & 1,60 & $1,03-2,35$ \\
\hline Acoso sexual & 4 & & & & & & & & & \\
\hline No & $(23,3)$ & & $(36,8)$ & & $(12,1)$ & & $(24,7)$ & & $(17,8)$ & \\
\hline Sí & 0,89 & $0,54-1,39$ & 1,21 & $0,90-1,55$ & 2,15 & $1,35-3,21$ & 1,29 & $0,88-1,79$ & 0,98 & $0,58-1,56$ \\
\hline Violencia sexual** & 1 & & & & & & & & & \\
\hline No & $(23,4)$ & & $(35,6)$ & & $(12,4)$ & & $(25,3)$ & & $(17,3)$ & \\
\hline Sí & 0,83 & $0,47-1,35$ & 1,49 & $1,12-1,84$ & 2,11 & $1,30-3,19$ & 1,14 & $0,74-1,64$ & 1,10 & $0,65-1,77$ \\
\hline Daño-asalto y robo de propiedad & 2 & & & & & & & & & \\
\hline No & $(23,9)$ & & $(36,9)$ & & $(12,6)$ & & $(23,9)$ & & $(15,3)$ & \\
\hline Sí & 0,74 & $0,44-1,17$ & 1,26 & $0,96-1,58$ & 1,98 & $1,26-2,94$ & 1,49 & $1,05-1,99$ & 1,93 & $1,28-2,73$ \\
\hline Observó agresión a conocido & 3 & & & & & & & & & \\
\hline No & $(23,2)$ & 4 & $(38,0)$ & & $(13,5)$ & & $(25,3)$ & & $(16,5)$ & \\
\hline Sí & 0,51 & $0,16-1,37$ & 1,74 & $1,18-2,17$ & 4,25 & $2,64-5,68$ & 1,99 & $1,21-2,77$ & 3,30 & $2,04-4,47$ \\
\hline
\end{tabular}

* Se informan razones de prevalencias ajustadas por edad-escolaridad y sexo con sus respectivos intervalos de confianza al $95 \%$.

Las cifras entre paréntesis son las prevalencias ajustadas en el grupo de referencia.

** Incluye besos-caricias-manoseo y violación sexual.

Fuente: encuestas aplicadas a asistentes de organizaciones e instituciones de, o enfocadas a, bisexuales, lesbianas

y homosexuales, Ciudad de México, 2001.

tionario tengan más dificultades para enfrentar el prejuicio y la exclusión hacia su orientación sexual, debido a que no cuentan con redes sociales de apoyo, y por tanto tenga mayor probabilidad de presentar daños a la salud, de ser así, las asociaciones identificadas en este estudio probablemente sean una subestimación de la relación de la violencia y la discriminación con los daños a la salud en la población bisexual, lesbiana y homosexual. Por ejemplo, entre la población bisexual, lesbiana y homosexual estudiada las prevalencias de niveles altos de homofobia internalizada (medida con la escala de Martin y Dean 20) y de ideación suicida fueron más altas entre los sujetos que nunca habían asistido a organizaciones sociales de los bisexuales, las lesbianas y los homosexuales (36,6\% y $39,5 \%$, respectivamente), en comparación a aquellos que sí frecuentaban esas organizaciones $(27,2 \%$ y $33,0 \%, \mathrm{p}<0,01)$.

El instrumento que se utilizó para recopilar la información también puede limitar los al- cances del estudio, pues los cuestionarios están sujetos a diferentes sesgos, por ejemplo, los individuos que no aceptan su orientación sexual es probable que no informen de todas las agresiones que han sufrido como un mecanismo para negar su homosexualidad, lo que los lleva a minimizar los hechos 7, produciéndose así una subestimación de la frecuencia de violencia. Para seleccionar los daños a la salud que se estudiarían se realizó una revisión de la literatura, este procedimiento puede determinar una limitante: que los problemas de salud identificados en otras poblaciones de los bisexuales, las lesbianas y los homosexuales (sobre todo de países industrializados) no sean los mismos que presenten los bisexuales, las lesbianas y los homosexuales de países como México. En futuras investigaciones será necesario utilizar técnicas cualitativas de investigación con el fin de conocer la percepción y vivencia de los problemas de salud de los bisexuales, las lesbianas y los homosexuales de México. Cabe 
acotar que las escalas que se utilizaron para la definición de los casos (alcoholismo, trastornos mentales comunes, ideación suicida e intento de suicidio) sólo proporcionan diagnósticos presuntivos, es decir, no son juicios clínicos definitivos. Finalmente, debido a la naturaleza transversal del estudio no se tiene certeza de que la exposición (la violencia y la discriminación) se haya presentado antes del evento (los daños a la salud), lo que limita la posibilidad de hacer conclusiones contundentes a partir de las asociaciones que se observaron en el estudio. A pesar de que algunos de los hallazgos son consistentes con lo informado por otros autores, asimismo las asociaciones que se observaron son congruentes con el conocimiento existente.

En la muestra de los bisexuales, las lesbianas y los homosexuales de la Ciudad de México las tasas de ideación suicida e intento de suicidio fueron de $39,0 \%$ y $15,0 \%$; mientras que en una encuesta representativa de la población urbana de México se informaron prevalencias de ideación suicida de $6,3 \%$ a $18,2 \%$ y de ideación suicida de $0,9 \%$ a $2,0 \%$, según el indicador que se utilice 21. En los adolescentes bisexuales, lesbianas y homosexuales (menores de 19 años) que encuestamos las prevalencias de ideación e intento de suicidio fueron $60,3 \%$ y $26,1 \%$, respectivamente, mientras que en los adultos bisexuales, lesbianas y homosexuales (de 19 años y más) las tasas fueron $35,7 \%$ y $13,7 \%$. En una revisión 22 de varios estudios sobre ideación e intento de suicidio en diferentes poblaciones de México se encontró que las prevalencias de ideación suicida en adolescentes variaron de $1,7 \%$ a $40,7 \%$ y en poblaciones adultas de $5,7 \%$ a $10,1 \%$; respecto a los intentos de suicidio en adolescentes van de $1,4 \%$ a $10,1 \%$ y en adultos de $3,0 \%$ a $6,1 \%$. Comparando los resultados de las diferentes fuentes, se puede apreciar que estos problemas de salud son más prevalentes en los bisexuales, las lesbianas y los homosexuales.

En la población total de los bisexuales, las lesbianas y los homosexuales de la Ciudad de México la prevalencia de trastornos mentales comunes fue de $27,0 \%$, la cual es similar a la reportada en otras poblaciones de América Latina. Utilizando el Self Reporting Questionnaire, Ludermir \& Lewis 23 encontraron que la prevalencia de trastornos mentales comunes en una ciudad de Brasil (Olinda) fue de 34,0\%; mientras que Araya et al. 24 notificaron una tasa de $26,7 \%$ para la Ciudad de Santiago en Chile, aunque los últimos consideraron como herramienta diagnóstica el Revised Clinical Interview Schedule. En una encuesta representativa de las zonas urbanas de México se informó que el $28,6 \%$ de la población alguna vez en la vida había presentado trastornos mentales 25 .

Para diagnosticar alcoholismo en los bisexuales, las lesbianas y los homosexuales de la Ciudad de México se utilizó la escala AUDIT 18, obteniéndose que en los varones bisexuales y homosexuales la frecuencia de alcoholismo fue de $17,0 \%$ y en las mujeres bisexuales y lesbianas de $21,0 \%$; con la misma escala en otras poblaciones de México se ha encontrado que la prevalencia de alcoholismo en los varones oscila entre $28,0 \%$ y $43,0 \%$, mientras que en las mujeres ha variado de $3,6 \%$ a $7,0 \% 26$, puede observarse que en los varones bisexuales y homosexuales encuestados la frecuencia de alcoholismo fue menor a la observada en otros varones; sin embargo, un hallazgo alarmante es que la frecuencia de alcoholismo en las mujeres bisexuales y lesbianas encuestadas fue hasta siete veces mayor a la reportada en otros grupos de mujeres.

A partir de una revisión de estudios sobre conducta suicida realizados en México, Gómez \& Borges 27 concluyeron que, en comparación con los hombres, las mujeres con mayor frecuencia intentan suicidarse, lo cual concuerda con las diferencias por sexo observadas en la población bisexual, lesbianas y homosexual que entrevistamos. De igual modo, respecto a los varones bisexuales y homosexuales, en las mujeres bisexuales y lesbianas de la Ciudad de México fue más alta la prevalencia de trastornos mentales comunes. Este patrón de acuerdo al sexo también fue observado en Santiago, Chile 24. Lo anterior contrasta con el hecho de que más varones bisexuales y homosexuales de la Ciudad de México fueron víctimas de ofensas verbales y agresión física, lo cual ha sido un hallazgo consistentemente notificado en la literatura 28. Aunque los estimadores notificados en este artículo derivaron de modelos de regresión logística en los que se incorporó al sexo como confusor potencial, estas discrepancias hacen necesario que en el futuro se analice, en hombres y mujeres por separado, el efecto que tienen en la salud mental la violencia y la discriminación por homofobia.

En la población de los bisexuales, las lesbianas y los homosexuales que estudiamos existió una clara asociación de algunas formas de violencia con indicadores de daños a la salud, así, por ejemplo, dos formas de violencia (violencia física y observar como agredieron a un conocido) se asociaron con mayor riesgo de presentar cuatro de los cinco indicadores de salud mental. Desde otra perspectiva, puede observarse que el alcoholismo y la ideación suicida 
se relacionaron con tres formas de violencia, los trastornos mentales comunes se asociaron con cuatro formas de violencia y el intento de suicidio se vinculó con cinco formas de violencia. Este hallazgo es consistente con lo observado en investigaciones realizadas en países industrializados 10,11. Distintos procesos pueden explicar la asociación entre violencia y daños a la salud observada en la población de los bisexuales, las lesbianas y los homosexuales. Algunos efectos de la violencia sobre la salud son inmediatos, visibles y evidentes, por ejemplo, una lesión o herida como resultado de agresión física o la pérdida de algún objeto por robo o asalto; desde el punto de vista psicológico, una de las reacciones evidentes que puede atribuirse directamente a la violencia es el estrés postraumático 29 .

Aunque algunas de las repercusiones de la violencia en los bisexuales, las lesbianas y los homosexuales son similares a la de cualquier víctima de violencia, los primeros pueden experimentar, debido al prejuicio contra la homosexualidad, algunas reacciones que no presentarán otras víctimas. Las víctimas de violencia tienden a buscar explicaciones de por qué fueron agredidas, en la búsqueda de explicaciones las víctimas frecuentemente se culpan a sí mismas de la violencia que recibieron, fenómeno que ha sido denominado auto-culpa (selfblame), la cual puede ser elaborada de dos formas: (a) auto-culpa caracterológica, en la que la persona identifica que algún rasgo o cualidad de su personalidad es la responsable de la agresión o (b) auto-culpa conductual, en la cual la víctima evalúa que alguna conducta o comportamiento suyo fue el origen de la violencia que recibió. La auto-culpa caracterológica puede reactivar o reforzar una auto-imagen negativa y provocar una sensación de que la persona merecía la agresión; mientras que en la auto-culpa conductual los individuos modificarán aquellos comportamientos que consideran dieron origen a la agresión, con lo cual consideran que tienen mayor control sobre los eventos y reducen así su percepción de vulnerabilidad 30,31,32,33. Se puede especular, entonces, que cuando los bisexuales, las lesbianas y los homosexuales son víctimas de violencia se culparán a sí mismos de la agresión que recibieron, ubicando como causa de la violencia sus pensamientos y deseos homoeróticos (auto-culpa caracterológica) o las expresiones de atracción o afecto a personas del mismo sexo (autoculpa conductual). Cuando los individuos atribuyen la violencia a rasgos de su personalidad (auto-culpa caracterológica) se producen cambios en su auto-imagen, comenzándose a per- cibirse a sí mismos de forma negativa, estos cambios se asocian con depresión, baja autoestima, autocrítica y sentimientos de que la persona merecía la agresión que recibió 30,31,32,33,34. Los bisexuales, las lesbianas y los homosexuales pueden evaluar que las agresiones que sufrieron fueron debidas a su orientación sexual, con lo cual se reforzará en ellos la creencia de que la homosexualidad es una condición denigrante que merece ser castigada, es decir, se incrementarán sus niveles de homofobia internalizada, lo cual, a su vez, conducirá a distintos niveles de sufrimiento mental 35. Además, cuando los individuos se culpan a sí mismos de la agresión que recibieron también desarrollan una percepción de vulnerabilidad única, en la que perciben que tienen mayor riesgo de sufrir un evento negativo en comparación con otras personas, esta percepción de vulnerabilidad única se asocia con depresión, baja auto-estima, ansiedad, distrés, miedo y ansiedad; los sentimientos de vulnerabilidad son resultado de que las víctimas comienzan a ver al mundo como algo desconocido, amenazante y que no puede controlarlo, lo que provoca en ellas una fuerte desconfianza en los demás 34 .

Es importante resaltar que las personas que presenciaron que algún conocido fue violentado, también presentaron prevalencias más altas de ideación suicida, intento de suicidio, trastornos mentales comunes y el alcoholismo, lo que indica que los efectos negativos de la violencia no sólo los presentan las personas que la sufren de forma directa. Se sabe que las personas que conocen a una víctima perciben que son más vulnerables de sufrir violencia en el futuro, especialmente cuando la persona percibe que es similar a la víctima que conoce, además de que el sentimiento de vulnerabilidad se asocia con miedo y ansiedad 31,33,34. Asimismo, cuando un individuo observa que violentan a otra persona por una característica que él comparte, recibe el mensaje de que él potencialmente también está en riesgo de sufrir la misma agresión lo cual puede generar ansiedad 36.

A diferencia de la violencia, existieron pocas asociaciones entre discriminación y daños a la salud. El menor efecto de la discriminación respecto a la violencia puede ser debida a que la primera, por lo regular, se da dentro de un contexto institucional, lo cual puede representar un límite de las prácticas discriminatorias por parte de los empleados. Esta situación puede hacer que los bisexuales, las lesbianas y los homosexuales conciban los actos de discriminación como formas de prejuicio que pueden ser fácilmente denunciadas o castigadas, es de- 
cir, los bisexuales, las lesbianas y los homosexuales pueden percibir mayor control sobre esos eventos. Los mecanismos que pueden explicar las pocas relaciones que observamos entre discriminación y salud pueden ser similares a los que ya se describieron para el caso de la violencia.

Algunos de los hallazgos de nuestro estudio son susceptibles de ser retomados para la formulación de políticas y programas de salud. Hasta ahora, los programas de salud de los gobiernos latinoamericanos que han abordado problemas específicos de la población bisexual, lesbiana y homosexual se han restringido a la prevención de la infección de VIH/SIDA entre hombres que tienen sexo con otros hombres. Estas acciones en gran medida han sido producto de la magnitud del problema y no porque se reconozca la existencia de estos grupos. Sin embargo, en esta investigación se ha evidenciado que además del VIH/SIDA, los bisexuales, las lesbianas y los homosexuales presentan otros problemas de salud, por ejemplo, un tercio de la población estudiada presentó sintomatología indicativa de trastornos mentales comunes y entre las mujeres bisexuales y lesbianas fue elevada la frecuencia de alcoholismo. Por lo tanto, se requiere que las instituciones gubernamentales amplíen los programas de bienestar y salud dirigidos a los bisexuales, las lesbianas y los homosexuales.

La discriminación y la violencia interpersonal que sufren los bisexuales, las lesbianas y los homosexuales son un problema que debe ser resuelto, en tanto que representan una limita- ción del goce pleno de derechos humanos de esta población. A lo anterior, hay que agregar que en nuestro estudio documentamos que ambas expresiones del prejuicio se relacionaron con mayor riesgo de presentar daños a la salud. Entre los bisexuales, las lesbianas y los homosexuales encuestados una de las formas de violencia más frecuente fue la verbal. El lenguaje es uno de los vínculos más importantes por el cual se reproduce el sistema de géneros, por lo que se requiere que se promueva el desuso de términos peyorativos o que denigren la homosexualidad. Esta tarea es indispensable para cambiar las representaciones y los significados asociados con homosexualidad. Asimismo, es necesario alentar la denuncia de la violencia y la discriminación que sufren los bisexuales, las lesbianas y los homosexuales, lo cual contribuiría al reconocimiento social e institucional de la desventaja social en la que se encuentran los bisexuales, las lesbianas y los homosexuales. En esta área las organizaciones civiles de bisexuales, lesbianas y homosexuales tendrán un papel fundamental. De igual modo, se requiere que las instituciones encargadas de la impartición de justicia sean más sensibles a la problemática de esta población. Las formas más frecuentes de discriminación que observamos fueron la detención, extorsión o amenaza de policías, la discriminación laboral y el maltrato de empleados de servicios, esto indica que se requiere que los prestadores de servicios públicos y privados reciban capacitación para eliminar el trato discriminatorio. 


\section{Resumen}

Los objetivos del estudio fueron estimar la frecuencia de daños a la salud mental (percepción del estado de salud, ideación suicida, intento de suicidio, trastornos mentales comunes y alcoholismo) en bisexuales, lesbianas y homosexuales de la Ciudad de México y analizar la posible relación de la violencia y la discriminación con la salud mental de dicha población. Se aplicó un cuestionario a 506 bisexuales, lesbianas y homosexuales de la ciudad de México para investigar si habían sufrido, debido a su orientación sexual, discriminación y violencia. Las prevalencias fueron: ideación suicida 39,0\%, intento de suicidio $15,0 \%$, trastornos mentales comunes $27,0 \%$ y alcoholismo 18,0\%. En las mujeres lesbianas y bisexuales se observó una prevalencia de alcoholismo siete veces mayor a la observada en otras mujeres. La discriminación se relacionó con el intento de suicidio y los trastornos mentales comunes, mientras que la violencia fue un factor de riesgo para la ideación suicida, el intento de suicidio, los trastornos mentales comunes y el alcoholismo; por lo cual se requiere implementar políticas y programas tendientes a revertir esta situación.

Violencia; Prejuicio; Salud Mental; Homosexualidad

\section{Colaboradores}

L. Ortiz-Hernández disenó el estudio, coordinó el trabajo de campo y realizó la estructura del artículo. M. I. G. Torres colaboró con la depuración de la base de datos, en el análisis estadístico y discusión de resultados.

\section{Agradecimientos}

Agradecemos a las siguientes organizaciones e instituciones por su valiosa colaboración para la realización del proyecto: Acción Humana por la Comunidad, Ave de México, Café Virreinas, Centro de Capacitación y Apoyo Sexológico Humanista, CEDART "Frida Kahlo", Clínica Especializada Condesa para la Prevención y Atención en VIH/SIDA, El Closet de Sor Juana, Fraternidad Gay, Fundación Mexicana para la Lucha contra el SIDA, Génesis Grupo Cristiano Ecuménico, Grupo de Madres Lesbianas, Hadas, Iglesia de la Comunidad Metropolitana, Letra S, Milk México, Módulo de Atención, Orientación y Quejas Ciudadanas de la Diputada Enoe Uranga, Musas de Metal.

\section{Referencias}

1. Comisión Ciudadana Contra los Crímenes de Odio por Homofobia. Informe de crímenes 2000. http:// www.cogailes.org/ilga/docs.pdf/ccccoh00def.pdf (accedido el 20/Feb/2004).

2. Gay and Lesbian Medical Association/LGBT Health Experts. Healthy people 2010 companion document for lesbian, gay, bisexual, and transgender (LGBT) health. San Francisco: Gay and Lesbian Medical Association; 2001.

3. Dean L, Meyer IH, Robinson K, Sell RL, Sember R, Silenzio V, et al. Lesbian, gay, bisexual, and trasgender health: findings and concerns. Journal of the Gay and Lesbian Medical Association 2000; 4:10151.

4. Carrier JM. Cultural factors affecting urban Mexican male homosexual behavior. Arch Sex Behav 1976; 5:103-24.

5. World Health Organization. World report on violence and health. Geneva: World Health Organization; 2002.

6. Herek GM. Hate crimes against lesbian and gay men. Issues for research and policy. Am Psychol 1989; 44:948-55.

7. Garnets L, Herek GM, Levy B. Violence and victimization of lesbians and gay men: mental health consequences. In: Herek GM, Berrill KT, editors. Hate crimes. Confronting violence against lesbians and gay men. Newbury Park: Sage Publications; 1992. p. 207-26

8. Vallejo-Aguilar OJ, Navarrete-Navarro S, del RioChiriboga C, Ávila-Figueroa C, Santos-Preciado JI. El trabajador de salud y la consejería sobre el VIH y SIDA. Salud Pública Méx 1995; 37:636-42.

9. Krieger N, Sydney S. Prevalence and health implications of anti-gay discrimination: a study of black and white women and men in the cardia cohort. Int J Health Serv 1997; 27:157-76.

10. Herek GM, Gillis JR, Cogan JC. Psychological sequelae of hate crime victimization among lesbian, gay, and bisexual adults. J Consult Clin Psychol 1999; 67:945-51.

11. Hershberger SL, D'Augelli AR. The impact of victimization on the mental health and suicidaly of lesbian, gay, and bisexual youths. Dev Psychol 1995; 31:65-74.

12. Martin JL, Dean L. Developing a community sample of gay men for a epidemiologic study of AIDS. Am Behav Sci 1990; 33:546-61.

13. Secretaría de Salud. Ley general de salud. México DF: Editorial Porrúa; 2000.

14. Klein F. The need to view sexual orientation as a multivariable dynamic process: a theoretical perspective. In: McWhirter DP, Sanders SA, Machover J, editors. Homosexuality/heterosexuality. Concepts of sexual orientation. New York: Oxford University Press; 1990. p. 277-82.

15. Secretaría de Salud/Instituto Mexicano de Psiquiatría. Encuesta nacional de adicciones. México DF: Secretaría de Salud/Instituto Mexicano de Psiquiatría; 1998.

16. Romero MR, Medina-Mora ME. Validez del Cuestionario General de Salud, para detectar psicopatología en estudiantes universitarios. Salud Mental 1987; 10:90-7. 
17. Goldberg DP. The detection of psychiatric illness by questionnaire. New York: Oxford University Press; 1972.

18. Organización Mundial de la Salud. AUDIT. Prueba de identificación de trastornos derivados del consumo de alcohol: pautas de uso en la atención primaria de la salud. Geneva: Organización Mundial de la Salud; 1992.

19. Zhang J, Yu KF. What's the relative risk? A method of correcting the odds ratios in cohort studies of common outcomes. JAMA 1998; 280:1690-1.

20. Meyer IH. Minority stress and mental health in gay men. J Health Soc Behav 1995; 36:38-56.

21. Medina-Mora ME, Rascón ML, Tapia R, Mariño MC, Juárez F, Villatoro J, et al. Trastornos emocionales en población urbana mexicana: resultados de un estudio nacional. Anales del Instituto Mexicano de Psiquiatría 1992; 3:48-55.

22. Mondragón L, Borges G, Gutiérrez R. La medición de la conducta suicida en México: estimaciones y procedimientos. Salud Mental 2001; 24:4-15.

23. Ludermir AB, Lewis G. Links between social class and common mental disorders in Northeast Brazil. Soc Psychiatry Psychiatr Epidemiol 2001; 36:101-7.

24. Araya R, Rojas G, Fritsch R, Acuña J, Lewis G. Common mental disorders in Santiago, Chile. Br J Psychiatr 2001; 178:228-33.

25. Medina-Mora ME, Borges G, Lara C, Benjet C, Blanco J, Fleiz C, et al. Prevalencia de trastornos mentales y uso de servicios: resultados de la Encuesta Nacional de Epidemiología Psiquiátrica en México. Salud Mental 2003; 26:1-16.

26. Medina-Mora E, Carreño S, De la Fuente JR. Experience with the Alcohol Use Disorders Test (AUDIT) en Mexico. In: Galanter M, editor. Recent developments in alcoholism. v. 1. New York: Plenum Press, 1998. p. 383-96.
27. Gómez C, Borges G. Los estudios que se han hecho en México sobre la conducta suicida: 19661994. Salud Mental 1996; 19:45-55.

28. Ortiz L, Granados JA. Violencia hacia bisexuales, lesbianas y homosexuales de la Ciudad de México. Rev Mex Sociol 2003; 3:265-303.

29. Asociación Americana de Psiquiatría. DSM-IV. Manual diagnóstico y estadístico de los trastornos mentales. Madrid: Masson; 1998.

30. Miller DT, Porter CA. Self-blame in victims of violence. J Soc Issues 1983; 39:139-52.

31. Hanson I, Greenberg MS, Hymer S. Describing the crime victim: psychological reactions to victimization. Prof Psychol Res Pr 1987; 18:299-315.

32. Peterson C, Seligman MEP. Learned helplessness and victimization. J Soc Issues 1983; 39:103-16.

33. Janoff-Bulman R, Frieze IH. A theoretical perspective for understanding reactions to victimization. J Soc Issues 1983; 39:1-17.

34. Perloff LS. Perceptions of vulnerability to victimization. J Soc Issues 1983; 39:41-61.

35. Ortiz L. La relación entre opresión y enfermedad en bisexuales, lesbianas y homosexuales de la ciudad de México [Tesis de Maestría]. México DF: Universidad Autónoma Metropolitana Xochimilco; 2003.

36. Gonsiorek JC. Mental health issues of gay and lesbian adolescents. J Adolesc Health Care 1988; 9:114-22

Recibido el 02/Ago/2004

Versión final presentada el 21/Dic/2004

Aprobado el 23/Dic/2004 